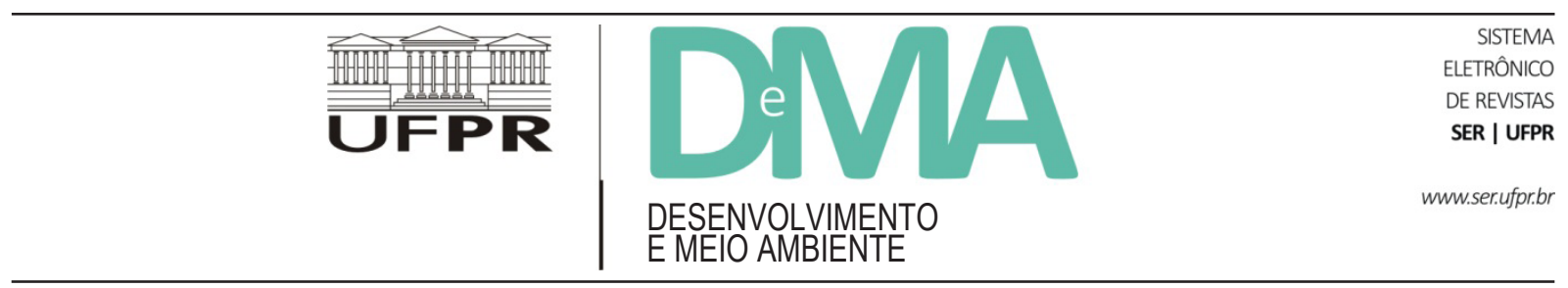

\title{
Alternativas locacionais em Avaliação de Impacto Ambiental de rodovias mineiras
}

\section{Location Alternatives in Environmental Impact Assessment of Roads in Minas Gerais State}

\author{
Ana Heloisa Vilela FERNANDES ${ }^{1}$, Maiko de Ávila CASSIANO ${ }^{1}$, Thales Cardoso Saraiva GUIMARÃES ${ }^{1}$, \\ Maria Rita Raimundo e ALMEIDA ${ }^{1^{*}}$ \\ ${ }^{1}$ Universidade Federal de Uberlândia (UFU), Uberlândia, MG, Brasil. \\ *E-mail de contato: mrralmeida@ufu.br
}

Artigo recebido em 22 de julho de 2017, versão final aceita em 14 de novembro de 2017.

RESUMO: A proposição de alternativas mais viáveis ambientalmente é considerada um dos princípios de boas práticas da Avaliação de Impacto Ambiental (AIA). Sem um estudo de alternativas, a AIA pode se reduzir à proposição de medidas mitigadoras de impactos que poderiam ser evitados. Apesar do crescente interesse acadêmico, ainda são poucos os estudos que trataram das alternativas locacionais, sobretudo no setor de transporte rodoviário. $\mathrm{O}$ presente trabalho objetiva avaliar como a consideração de alternativas tem sido desenvolvida dentro dos processos de AIA de rodovias tramitados no órgão ambiental estadual de Minas Gerais. A seleção dos estudos de caso visou encontrar os processos de licenciamento ambiental de rodovias (implantação ou duplicação) tramitados no órgão ambiental do Estado de Minas Gerais e baseados em Estudos de Impacto Ambiental/Relatório de Impacto Ambiental (EIA/Rima) e, então, comparar os critérios utilizados para a seleção de alternativas destes casos com as boas práticas preconizadas na literatura. Deste modo, foram encontrados no Sistema Integrado de Informações Ambientais (SIAM) 10 casos no Estado de Minas Gerais, dos quais três necessitaram da elaboração de EIA/Rima e, destes, todos possuíam o estudo de alternativas locacionais. A partir da literatura, foi encontrada uma série de critérios gerais a serem abordados e falhas comuns que necessitam ser evitadas durante a elaboração das alternativas. Ao compará-los com os casos, obteve-se um resultado preocupante, visto que, mesmo cumprindo alguns critérios de boas práticas, todos os estudos de alternativas apresentaram-se de forma precária, com informações sem detalhamento e falhas que poderiam ter sido evitadas. Sendo assim, pode-se dizer que, no âmbito do órgão ambiental licenciador do Estado de Minas Gerais, o estudo de alternativas locacionais para rodovias é considerado frágil, não havendo a profundidade necessária para avaliar a viabilidade ambiental.

Palavras chave: licenciamento ambiental; avaliação de impacto ambiental; alternativas locacionais. 
ABSTRACT: Alternatives studies are considered as one of the best practice principles of Environmental Impact Assessment (EIA). Without an alternative study, EIA is reduced to proposition of mitigating measures of impacts that could have been avoided. Despite the growing academic interest, there are still few studies dealing with location alternatives, especially in the road transport sector. Therefore, the present research aims to evaluate how the consideration of alternatives has been developed within the EIA processes of roads processed in environmental agency of Minas Gerais state. The selection of case studies aimed to find environmental licensing processes of roads processed in Minas Gerais state and based on Environmental Impact Studies (EIS) and then compare criteria used for the selection of alternatives of those cases with the best practices recommended in the literature. In Integrated Environmental Information System (Sistema Integrado de Informações Ambientais - SIAM), 10 cases were found in overall Minas Gerais state, of which three required EIS elaboration, and all of them had study of location alternatives. From the literature, a series of general criteria to be addressed and common failures that need to be avoided during the elaboration of alternatives studies was found. When comparing them with the cases, a worrying result appeared, since all alternatives studies presented themselves in a precarious way, with information without detailing and failures that could have been avoided, while meeting some good practice criteria. Thus, it can be said that in environmental agency of Minas Gerais state, location alternatives study for roads is considered fragile, and there is no necessary and due consideration of this essential factor in decision making on environmental viability.

Keywords: environmental licensing; environmental impact assessment; location alternatives.

\section{Introdução}

A Avaliação de Impacto Ambiental (AIA) é um dos instrumentos mais amplamente empregado no mundo para a verificação dos futuros efeitos da ação humana sobre o meio. Introduzida em 1969 nos Estados Unidos, a AIA compreende o processo de identificar, prever, avaliar e mitigar os efeitos relevantes de natureza biofísica, social e outros efeitos de atividades ou projetos de desenvolvimento, antes que decisões importantes sejam tomadas (IAIA, 1999). A partir de sua introdução, a AIA transformou-se em uma ferramenta internacionalmente aceita e estabelecida (Jayet al., 2007; Sánchez, 2008), constituindo o instrumento de verificação dos efeitos da ação antrópica sobre o meio mais amplamente empregado no mundo: 191 dos 193 membros das Nações Unidas têm algum regulamento, legislativo ou não, que refira o uso da AIA (Morgan, 2012).

A introdução da AIA no Brasil veio após a reunião sobre meio ambiente promovida pela $\mathrm{ONU}$, em Estocolmo (1972), em que os efeitos negativos da ação do homem sobre o ambiente passam a ser considerados e reconhece-se a necessidade de controlá-los. Esta introdução ainda envolve as instituições e agências multilaterais de desenvolvimento que passaram a adotar regulamentos e requisitos para a verificação dos efeitos ambientais negativos decorrentes dos projetos em financiamento.

A partir de então, reproduzindo um processo que se verifica em muitos países, a AIA passa para o corpo institucional brasileiro, primeiramente em alguns estados,até que, finalmente, é estabelecida pela Política Nacional de Meio Ambiente (Lei Federal $n^{0} 6.938$ de 1981) como instrumento de política ambiental. Posteriormente, passa a ser regulamentada pelas Resoluções Conama $n^{\circ} 01$ de 1986 e n$^{\circ} 237$ de 1997 e, em termos de competência, pela Lei Complementar $n^{\circ} 140$ de 2011, tendo sua utilização no âmbito do licenciamento ambiental de empreendimentos causadores de significativo impacto ambiental.

Apesar da experiência acumulada mundialmente, muitas críticas são feitas quanto à efetividade 
deste instrumento (conforme os trabalhos de Morgan, 2012; Almeida \& Montaño, 2017), amparadas, sobretudo, pelo distanciamento verificado entre teoria e prática (Lobos \& Partidário, 2014).

Entre os aspectos da AIA mais frequentemente criticados está a consideração de alternativas para o projeto (Popeet al., 2013). Segundo estudos realizados, a proposição de alternativas tecnológicas e locacionais é incipiente ou inexistente (Hickie \& Wade, 1998; Steinemann, 2001; Pinho et al., 2007; Kruopienè et al., 2009) ou abrange alternativas ambientalmente inviáveis (Zubair, 2001).

No Brasil, a partir de uma reflexão sobre a efetividade da AIA, o Ministério Público Federal (MPF, 2004) apontou as principais deficiências encontradas nos Estudos de Impacto Ambiental (EIA) e também em algumas etapas da AIA, sendo que entre estas deficiências estavam a ausência de proposição de alternativas, a apresentação de alternativas reconhecidamente inferiores à selecionada, a prevalência dos aspectos econômicos sobre os ambientais nas escolhas de alternativas e a comparação de alternativas a partir de base diferenciada. Assim, comumente, a análise de alternativas se restringe à avaliação de uma única alternativa, impedindo-se o confronto de opções no processo de análise e configurando um reducionismo da aplicação da AIA, que se torna reativa ao se restringir à identificação de medidas mitigadoras (Agra Filho et al., 2012).

A proposição de alternativas tecnológicas e locacionais, considerada por Paliwal (2006) como um dos princípios de melhores práticas da AIA, é fundamental na elaboração dos estudos de impacto ambiental, a fim de que opções mais viáveis ambientalmente sejam escolhidas. O exame de alternativas, já há quase duas décadas, é considerado como um dos Princípios Operacionais da AIA (IAIA, 1999) e a própria legislação brasileira especifica que sejam consideradas todas as alternativas tecnológicas e de localização de projeto submetidos a EIA/Rima (CONAMA, 1986).

Como o potencial de causar impacto depende da pressão que a tipologia do empreendimento exerce sobre os recursos naturais e da sensibilidade do local em que se pretende implantar o projeto, sem um estudo de alternativas, principalmente locacionais, a avaliação de impactos pode ficar reduzida apenas à proposição de medidas para remediar impactos que poderiam ser evitados se o local mais adequado houvesse sido escolhido. Para algumas tipologias de projetos, a discussão de alternativas locacionais é ainda mais relevante: este é o caso dos chamados empreendimentos lineares, como rodovias, nos quais a discussão de alternativas costuma ser utilizada principalmente para a seleção de traçado.

Rodovias são estruturas complexas que têm como objetivo principal servir como via de transporte terrestre para pessoas e cargas (Bandeira \& Floriano, 2004). As rodovias são atividades modificadoras do meio ambiente e que dependem de elaboração de Estudo de Impacto Ambiental e do respectivo Relatório de Impacto Ambiental (EIA/Rima) em seu processo de licenciamento (CONAMA, 1986). Isso porque seus impactos iniciam no planejamento, continuam na fase de implantação e construção, até a fase operacional, quando a qualidade de sua manutenção tem grandes implicações, sendo estes impactos relacionados a: ruídos, qualidade do ar, qualidade da água, processos erosivos, impactos sobre a fauna e a flora, aspectos estéticos e visuais, impactos econômicos e sociopolíticos (Bandeira \& Floriano, 2004). Assim, podem ser verificados complexos impactos ao meio físico, gerados em sua maioria na fase de construção, em decorrência da não correção das áreas utilizadas como bota-foras, aterros e caixas e jazidas de empréstimo (Omena \& Santos, 2008). Ainda, as atividades de empreen- 
dimentos lineares, como é o caso de rodovias, são consideradas potencialmente perigosas para a conservação da biodiversidade, principalmente, pela fragmentação da cobertura vegetal e pelo atropelamento de animais (Rangel \& Bueno, 2016).

No contexto brasileiro, as rodovias constituem o principal modal de transporte, sendo que o Estado de Minas Gerais tem a maior malha rodoviária do Brasil, equivalente a $16 \%$ de toda a malha viária existente no país: são $269.543 \mathrm{~km}$ de rodovias, sendo $7.689 \mathrm{~km}$ de rodovias federais, $23.663 \mathrm{~km}$ de rodovias estaduais e $238.191 \mathrm{~km}$ de rodovias municipais (Governo de Minas, 2017).

Reafirmando a importância da discussão de alternativas dentro do processo de AIA, em 2013, a Associação Brasileira de Entidades Estaduais de Meio Ambiente (ABEMA) criou o documento intitulado Novas propostas para o licenciamento ambiental no Brasil (ABEMA, 2013), onde identifica e propõe ações para vencer os desafios da aplicação deste instrumento no Brasil. Entre estes desafios estava a adoção do critério locacional e a consequente abordagem de alternativas para o projeto a ser licenciado. Duarte et al. (2017), ao avaliar a percepção dos praticantes de AIA no Brasil, encontraram que, dentro da categoria qualidade dos estudos de impacto ambiental, entre as percepções negativas estavam as deficiências relacionadas com o estudo de alternativas. Ainda, Fonseca et al. (2017), ao avaliarem a opinião de especialistas sobre as prioridades para as modificações do sistema de AIA no Brasil, apontaram que o fator locacional é importante para entender a significância dos impactos causados e definir a necessidade ou não do licenciamento e da AIA.

De uma forma mais aplicada à realidade de um órgão ambiental, Almeida e Montaño (2015) apontaram a falta de consideração de alternativas para o projeto ao avaliarem estruturalmente o sistema licenciador do Estado de Minas Gerais.

Para tentar solucionar esta situação, o distanciamento entre teoria e prática pode ser reduzido por meio da aplicação de procedimentos voltados para a avaliação do cumprimento dos objetivos da AIA. Para Agra Filho et al. (2007), a avaliação ex-post é considerada uma etapa imprescindível da avaliação de impacto ambiental por permitir que os resultados obtidos sejam continuamente analisados, proporcionando uma aprendizagem para os envolvidos no processo e, portanto, sendo importante para o aprimoramento contínuo desse instrumento.

Diante deste contexto, torna-se pertinente o objetivo do presente artigo de avaliar como estão sendo desenvolvidos os estudos de alternativas locacionais para a seleção de traçado de rodovias dentro dos processos de Avaliação de Impacto Ambiental desenvolvidos junto ao órgão ambiental do Estado de Minas Gerais.

\section{Metodologia}

A metodologia do presente trabalho baseia-se em uma abordagem qualitativa de investigação baseada na análise de conteúdo de documentos. Bardin (2011), recorrendo a Berelson, define análise de conteúdo como uma técnica de investigação que, por meio de uma descrição objetiva, sistemática e quantitativa do conteúdo manifesto das comunicações, tem por finalidade a interpretação destas mesmas comunicações. Complementando, a análise de conteúdo é uma dentre as diferentes formas de interpretar o conteúdo de um texto, adotando normas sistemáticas de extrair significados, por meio dos elementos mais simples do texto (Sá-Silva et al., 2009). Esta técnica tenta trazer maior rigor à análise (Bardin, 2011). 
Os documentos sujeitos à análise de conteúdo neste trabalho foram os Estudos de Impacto Ambiental (EIA) dos casos selecionados que constituem processos de licenciamento ambiental em âmbito do órgão ambiental estadual de Minas Gerais. Nos EIAs, foram de interesse as informações apresentadas no estudo de alternativas. $\mathrm{Na}$ descrição do estudo de alternativas do EIA, buscou-se elementos para avaliar o conjunto de indicadores selecionados.

Assim, as etapas de desenvolvimento no presente trabalho se dividiram em: i) seleção dos estudos de caso; ii) seleção dos critérios para análise do estudo de alternativas; e iii) comparação entre os critérios de melhores práticas e o estudo de alternativas dos casos selecionados.

\subsection{Seleção dos estudos de caso}

A seleção dos estudos de caso foi realizada com o uso da ferramenta de busca on-line do Sistema Integrado de Informações Ambientais (SIAM) do Estado de Minas Gerais e procurou encontrar os processos de licenciamento ambiental de rodovias onde a análise de viabilidade ambiental foi baseada na elaboração de EIA/Rima. Pelo fato de o SIAM se tratar de uma ferramenta de disponibilização de informações de processos de licenciamento em Minas Gerais, nem todos os processos tramitados no estado estão presentes na plataforma, especialmente aqueles realizados há mais tempo. Sendo assim, a pesquisa não adotou um recorte temporal e sim englobou todos os processos de licenciamento disponíveis no SIAM que atendiam ao objetivo da pesquisa, independente de quando tenham ocorrido.

Assim, no SIAM, utilizou-se como filtro de retorno de processos o código E-01-01-5, "Implantação ou duplicação de rodovias" (código este dado pela Deliberação Normativa n ${ }^{\circ} 74$ de 2004 do Con- selho Estadual de Meio Ambiente de Minas Gerais para os projetos de rodovias a serem implantadas ou duplicadas), aplicado na seleção "Atividade(s) do empreendimento". Esta procura resultou em 128 registros de empreendimentos, sendo que apenas 10 se referiam realmente à implantação de novos empreendimentos desta tipologia.

Em um segundo momento, nestes 10 processos foram buscados aqueles em que o processo de licenciamento contou com a elaboração do Estudo de Impacto Ambiental e do respectivo Relatório de Impacto Ambiental (EIA/Rima). Entre estes registros, três obras necessitaram da elaboração de EIA/ Rima, enquanto que o restante apresentou como estudo o Relatório de Controle Ambiental (RCA), que é um tipo de estudo de avaliação de impacto no contexto mineiro menos abrangente e mais simplificado que o EIA/Rima. Dos três processos que contaram com a elaboração de EIA/Rima, todos possuíam o estudo de alternativas locacionais, sendo, portanto, os estudos de caso aqui utilizados. $\mathrm{Na}$ Tabela 1 é apresentada a relação dos 10 processos de licenciamento de novas rodovias mineiras, bem como o empreendedor responsável, o número do processo e o tipo de estudo envolvido.

\subsection{Seleção dos critérios para análise do estudo de alternativas}

Para selecionar os critérios que a literatura recomenda que devam ser considerados ao se selecionar alternativas locacionais de empreendimentos, foi realizada uma busca por artigos, livros, teses e dissertações que abordassem estudos de alternativas em empreendimentos lineares, como é o caso das rodovias. A pesquisa foi feita em sites específicos de trabalhos acadêmicos e publicações do gênero, como Scielo, Google Acadêmico, Banco de teses 
TABELA1 - Processos de licenciamento de novas rodovias em Minas Gerais levantados por meio das informações do SIAM

\begin{tabular}{cccc}
\hline Empreendedor & Empreendimento & $\mathbf{N}^{\mathbf{0}}$ Processo & Tipo de estudo \\
\hline CBA* & Acesso de interligação ao complexo de mineração & $11110 / 2006$ & RCA \\
\hline DNIT** & Rodovia BR-352, trecho entre MG-188B e BR-146 & $20479 / 2010$ & RCA \\
\hline DNIT** & BR-265 & $03497 / 2007$ & RCA \\
\hline DNIT** & BR-265/Jacui - Altinópolis & $08899 / 2005$ & RCA \\
\hline DNIT** & BR-050, km 0,0 ao km 69 - MG & $\mathbf{1 9 7 5 4 / 2 0 0 9}$ & EIA/Rima \\
\hline DNIT** & MG-381 - subtrecho: km 450 (MG-020) - km 143,61 (BR-116/MG) & $\mathbf{0 1 3 2 3 / 2 0 0 7}$ & EIA/Rima \\
\hline DER-MG*** & Ligação Limeira do Oeste (Usina Coruripe) entre & MG-497 (Carneirinho) & RCA \\
\hline DER-MG*** & MG-341 - Trecho Piumhi - São Roque de Minas & $00390 / 2004$ & RCA \\
\hline DER-MG*** & MG-353 - Trecho de acesso rodoviário ao aeroporto regional da & $15908 / 2007$ & RCA \\
\hline Vale S/A & Zona da Mata & $\mathbf{1 0 2 1 4 / 2 0 1 0}$ & EIA/Rima \\
\hline $\begin{array}{l}\text { * Companhia Brasileira de Alumínio; ** Departamento Nacional de Infraestrutura de Transportes; *** Departamento de Estradas e Rodagem } \\
\text { do Estado de Minas Gerais. }\end{array}$
\end{tabular}

e dissertações da USP e Science Direct, além de revistas da área de AIA como a Environmental Impact Assessment Review e a Impact Assessment and Project Appraisal.

Nesta busca, não foram encontrados trabalhos específicos e que já propusessem um conjunto de critérios para avaliar estudos de alternativas e, muito menos, de alternativas lineares. Para exemplificar esta ausência de estudos, Duarte et al. (2017), ao realizarem um levantamento sobre a produção de artigos científicos sobre licenciamento ambiental com base em Avaliação de Impacto Ambiental (AIA) no Brasil, identificaram, especificamente sobre análise de alternativas, apenas estudos que apresentavam métodos e ferramentas de AIA para definir alternativas e não critérios/indicadores para propor e avaliar alternativas. No entanto, foram encontrados trabalhos que apontavam características de boas práticas que um processo de escolha de alternativas deveria ter. Um exemplo é o trabalho de Furlanetto (2012) que, em sua revisão de literatura, levantou diversos trabalhos que discutiam a questão de alternativas. Usando como referência o que estes trabalhos citados apresentavam sobre alternativas, foi possível criar critérios/indicadores para avaliar os estudos de caso.

Além dos trabalhos acadêmicos, ao propor estes critérios/indicadores foram levados em consideração: a Resolução Conama n ${ }^{\circ}$ 001/1986, que diz que o estudo de impacto ambiental deve seguir a diretriz de "Contemplar todas as alternativas tecnológicas e de localização de projeto, confrontando-as com a hipótese de não execução do projeto" (CONAMA, 1986); o livro de Sánchez (2008) que, ao propor um guia para análise técnica de estudos ambientais, apresentou itens a serem analisados no estudo de alternativas; e as orientações da International Association for Impact Assessment (IAIA) para a proposição de alternativas em projetos (IAIA, 2015).

Assim, foi proposto o conjunto de critérios apresentado na Tabela 2, que foram aplicados ao estudo de alternativas desenvolvido nos estudos de caso selecionados. Vale destacar que o conjunto de indicadores proposto pode ser aplicado ao estudo de alternativas de qualquer tipologia de empreendimento, não sendo específico para rodovias. 
TABELA 2- Critérios gerais utilizados para avaliação dos estudos de alternativas locacionais.

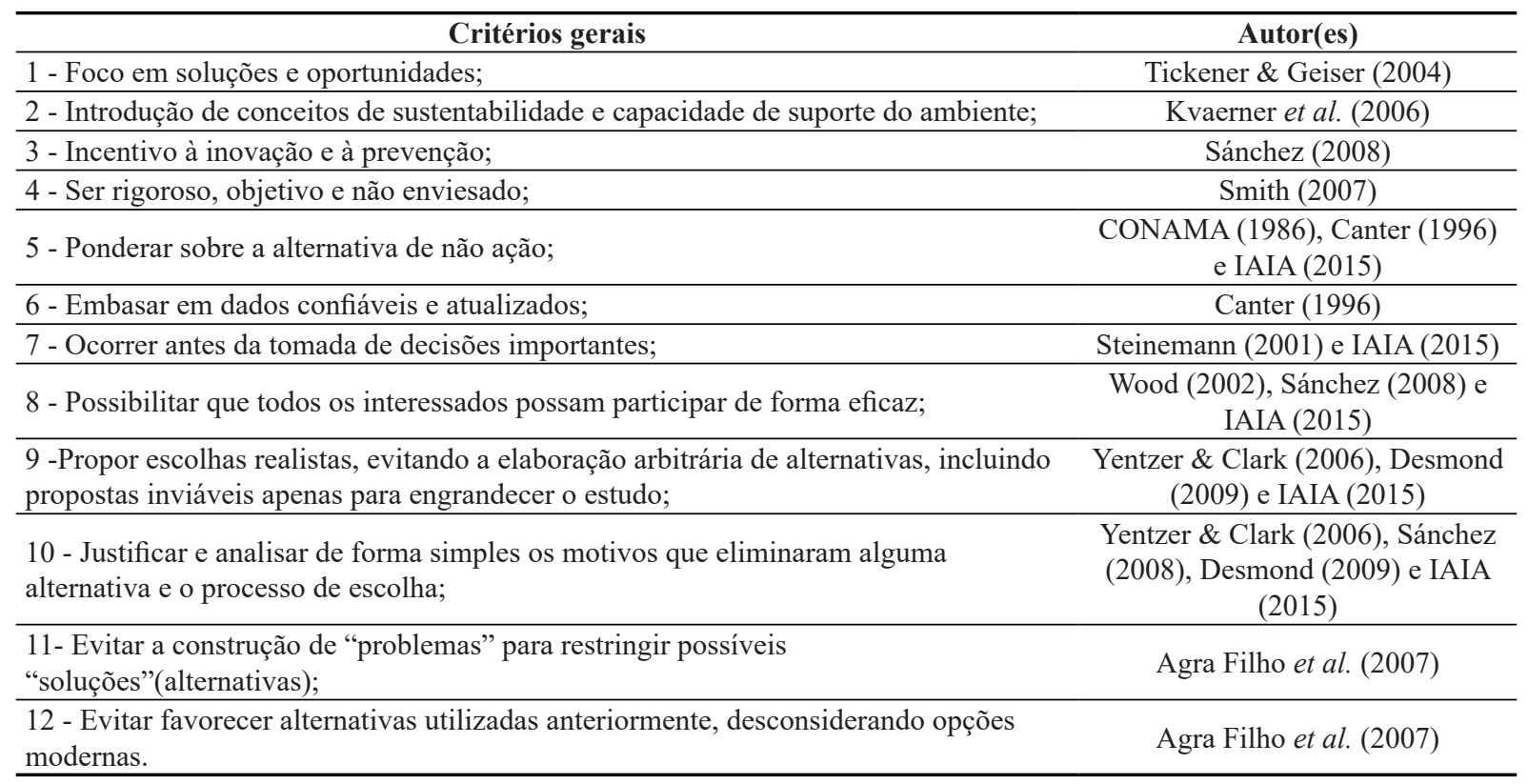

\subsection{Comparação entre os critérios de boas} práticas e o estudo de alternativas dos casos selecionados

Uma vez selecionados os estudos de caso e os critérios de boas práticas para a escolha de alternativas apresentados pela literatura, fez-se a comparação entre eles. Inicialmente, nos três casos selecionados, analisou-se o estudo de alternativas apresentado pelo EIA e levantou-se o procedimento utilizado na seleção da alternativa escolhida. Este procedimento foi avaliado perante os 12 critérios apresentados na Tabela 2. Cada critério de boas práticas foi avaliado em cumprido ou não cumprido.

\section{Resultados e discussão}

Nos resultados e discussões, primeiramente, é apresentada uma breve descrição de cada um dos três casos, das alternativas apresentadas no EIA/Rima e do procedimento de seleção de alternativas. Ao final, é mostrado o resultado de como cada critério de melhores práticas foi avaliado.

\subsection{O estudo de alternativas no Processo $01323 / 2007$}

O Processo 01323/2007 apresentava o EIA/ Rima solicitado pela Fundação Estadual do Meio Ambiente de Minas Gerais (FEAM) durante a etapa de Licença Prévia do Projeto de Ampliação da Capacidade e Modernização da Ligação Rodoviária entre Belo Horizonte e Governador Valadares Plano Funcional. O Programa de Ampliação do trecho da BR-381, com extensão nominal de 306,4 $\mathrm{km}$, previa obras de melhoramentos e duplicação na rodovia, tendo sido considerado de grande porte. No EIA, foram estudadas três alternativas de traçado 
com o objetivo de otimizar o custo operacional e a segurança na operação da rodovia, considerando juntamente as questões socioambientais. A seguir, serão brevemente descritas as alternativas, conforme as informações do EIA (CEC, 2007).

A alternativa I proposta instalar-se-ia predominantemente em áreas de pastagens, onde prevalecem fazendas e sítios voltados à criação de gado, buscando evitar a proximidade com as margens dos córregos para não gerar a degradação de ambientes naturais. Esta primeira possibilidade tinha por objetivo atingir o alto dos divisores dos córregos do Peixe e do Engenho, compreendendo os subtrechos 01 a 08 e incorporando parte da rodovia MG-129 (Itabira - BR-381) até o entroncamento com o acesso a Santa Maria do Itabira, na extensão total de $293 \mathrm{~km}$. Como ponto positivo, permitiria o aproveitamento com melhoramentos da via estadual existente, mas possuía uma altimetria que iria interferir negativamente em termos de desempenho operacional, apresentando rampas extensas e íngremes, com valores entre $6 \%$ e $7 \%$ em $12,8 \mathrm{~km}$. Não obstante, existia um trecho com necessidade de implantação de vários túneis e viadutos extensos, chegando estes a $1.800 \mathrm{~m}$, para transpassar os maciços rochosos e fundos de vale presentes. Além disso, havia o término das obras de ampliação da travessia de Nova Era (Obra Emergencial), a $5 \mathrm{~km}$ de proximidade, o que tornava mais atrativo investir na duplicação do segmento restante ao invés de iniciar um projeto com características desfavoráveis e altos custos. Quanto ao foco ambiental, essa alternativa indicou várias adequações do traçado para torná-lo viável.

Assim como a primeira alternativa, o segundo traçado proposto (alternativa II) foi planejado predominantemente em áreas de pastagens, onde prevaleciam fazendas e sítios voltados à criação de gado, buscando também evitar a proximidade com as margens dos córregos para não gerar a degra- dação de ambientes naturais e o seccionamento de corredores ecológicos. Esta proposta compreende os mesmos subtrechos referidos na alternativa I, acrescidos da Variante do Rio Santa Bárbara - subtrechos 09 e 10, com a extensão total de 295,89 km. Para o planejamento, previu-se uma alteração da diretriz geral da rodovia a acontecer entre o entroncamento com a rodovia estadual de acesso a Itabira e Nova Era, num intervalo de aproximadamente $45 \mathrm{~km}$. Entre as propostas nos subtrechos, encontravam-se áreas sensíveis em termos ambientais, como a presença de um talvegue consideravelmente acentuado e cercado por vegetação nativa, formando um amplo corredor ecológico. Para este caso, consideraram-se os fatores hidrológicos e hidráulicos da passagem e, buscando evitar o seccionamento do meio ambiente, indicou-se a implantação da obra em nível elevado. Além desta, o traçado apresentado também interferiria em outra faixa protegida por mata nativa e alguns afloramentos rochosos, desfragmentando área com reflorestamento de eucaliptos, o que gerou a necessidade de avaliação das condições de preservação ou proteção em ambos os episódios.

Por fim, a alternativa III referia-se a manter o segmento entre Belo Horizonte e o entroncamento com o acesso a Itabira, realizando obras de alargamento da rodovia atual e modificações localizadas internamente ou bem próximas da faixa de domínio já existente, estabelecida em 80,00 m de largura, simétrica em relação ao eixo da pista existente. A extensão final desta proposta é de $301,55 \mathrm{~km}$. Entre Antônio Dias e o Ribeirão Prainha, previu-se a implantação de uma via que não dependeria da via existente para o fluxo de tráfego no sentido Governador Valadares-Belo Horizonte. Tal opção foi tomada devido às características do relevo local, predominantemente montanhoso, o que resultou em um traçado com rampas elevadas. 
Neste processo, a alternativa I foi abandonada por aspectos técnicos e econômicos, pois propôs uma possibilidade com ganho operacional não significativo e custos inviáveis para operar durante sua vida útil. A alternativa II mostrou-se mais viável quando comparada à primeira, apresentando maior ganho operacional em resposta a um traçado moderno e por um custo de investimento consideravelmente inferior. A última alternativa, apesar de possibilitar menor custo de implantação e melhorar a segurança na pista já existente, não ofereceu quase nenhum ganho operacional necessitado por uma via de longa distância.

Assim, foi considerado que alternativa II obteve os melhores resultados tanto em capacidade como em velocidade operacional, custos econômicos e ideais condições de segurança para os usuários. Apresentou um novo traçado geométrico com $9,5 \mathrm{~km}$ a menos quando comparado ao percurso existente, possuindo características técnicas modernas, rampas fracas e médias, curvas amplas e, principalmente, evitando interferir em áreas urbanas. Entretanto, como a alternativa III referia-se ao alargamento de uma pista existente, obteve maior sucesso quanto aos níveis de segurança esperados, quando comparada à alternativa II. Sendo assim, a decisão tomada foi de se implantar a Variante do rio Santa Bárbara, em pista dupla - alternativa II, possibilitando almejar as melhorias necessárias ao corredor viário da BR-381, ocorrendo concomitantemente à alternativa III - rodovia atual, buscando reduzir os impactos negativos referentes à segurança. Foi considerado que unir as duas propostas ocasionaria um resultado mais adequado e benéfico, uma vez que, somadas, conseguem abranger as restrições à segurança na rodovia atual e resolver a problemática sobre capacidade de fluxo de longa distância. Porém, vale ressaltar que executar a alternativa II acarretaria em diminuição da qualidade ambiental no vale do rio Santa Bárbara, visto que desagregaria o equilíbrio dos aspectos naturais na região. Contudo, considerou-se, neste caso, o fato de que os benefícios sociais e econômicos intrínsecos ao projeto e os custos ambientais consequentes da instalação da proposta seriam assimilados.

\subsection{O estudo de alternativas no Processo $19754 / 2009$}

No Processo 19754/2009, o Departamento Nacional de Infraestrutura de Transporte (DNIT), objetivando a Licença Prévia, apresentou um EIA/ Rima sobre o Projeto de Restauração, Adequação e Duplicação da Rodovia BR-050, no subtrecho entre a Divisa GO/MG e o Entroncamento com as Rodovias BR-365/452/455/497, no Estado de Minas Gerais. A proposta visava atender a demanda de tráfego crescente (12.000 veículos/dia em 2006) com as devidas condições de segurança, sem precisar construir uma nova rodovia no trecho, e contou com duas alternativas locacionais.

Quanto às alternativas locacionais apresentadas no EIA (Escritório Técnico H. Lisboa da Cunha, 2009), foram consideradas algumas premissas e orientações para propor os traçados, visando minimizar os impactos socioambientais. Dentre estes pontos estavam: evitar ao máximo os ecossistemas sensíveis; contornar as áreas e utilizar a menor área necessária à supressão de vegetação (desmatamento); optar por áreas que já estejam mais degradadas/antropizadas; adotar menores trajetos; optar por técnicas construtivas que gerem as mínimas perturbações na paisagem e no funcionamento dos ecossistemas; e utilizar faixas existentes ou em licenciamento de outros empreendimentos. Entretanto, vale ressaltar que, no caso do trecho da BR-050, as variações locacionais não são realmente alternativas, 
mas pontos que devem ser evitados. O traçado propriamente dito, nos limites locacionais definidos, foi analisado em situações pontuais e considerando obstáculos naturais (relevo, trechos rochosos), localização de pontes e viadutos a duplicar, de acordo com o nível de gastos, de maior facilidade construtiva e ambientalmente menos impactantes, gerando assim a necessidade de planejar a pista alternando seus lados. Assim, foram apresentadas duas alternativas que auxiliaram nas justificativas para a definição do traçado, as quais serão apresentadas.

A primeira alternativa considera aspectos técnicos, econômicos e ambientais, buscando evitar, sempre que possível, significativos impactos sobre as áreas lindeiras à rodovia, minimizando gastos com desapropriação, volume de terraplenagem, supressão de remanescentes de vegetação nativa, entre outras interferências. Dessa forma, em certos pontos com devidas justificativas, fez-se necessário mudar o lado de implantação da nova pista. A obra, proposta em dois lotes e que poderia ser realizada tanto do lado esquerdo quanto do lado direito, foi projetada de forma a mesclar as duas possibilidades sempre que o ato conviesse.

Já a segunda alternativa trata da hipótese de não implantação do empreendimento, a qual seria uma escolha provisória caso fosse aceita, visto que a rodovia necessitaria de melhorias, em um curto espaço de tempo, devido a sua saturação de capacidade e posição estratégica em termos macrorregionais. Ademais, a manutenção da obra estava inclusa no Programa de Aceleração do Crescimento (PAC), lançado pelo Governo Federal, já possuindo viabilidade em termos econômicos. Como pontos negativos, a não realização das obras e serviços na rodovia acarretaria: tendência à saturação do tráfego; tendência à manutenção ou redução da produção agropecuária nos municípios servidos pela falta de condições adequadas de escoamento; degradação física pelo aceleramento do estado crítico do passivo ambiental e de processos erosivos instalados; crescente carreamento de sólidos e tendência à saturação dos atuais dispositivos de drenagem, com desdobramentos para novos focos erosivos, queda na qualidade das águas da rede hidrográfica regional e das bacias do Rio Grande e do Paranaíba, em longo prazo; dificuldades de acesso da população de Araguari aos serviços mais especializados (saúde, educação) disponíveis em Uberlândia, com uso intenso (ônibus semiurbanos a cada 30 minutos); não geração de emprego e renda pela não implantação e operação do empreendimento; e não promoção do desenvolvimento da economia nos Estados de Minas Gerais e Goiás, dentre outros fatores.

A alternativa I foi escolhida, considerando os aspectos negativos da segunda alternativa de não se fazer o empreendimento e visto que o traçado variável era a melhor opção para se adequar o projeto às questões ambientais, permitindo menores impactos sobre as áreas lindeiras, diminuindo custos com desapropriação, reduzindo volumes de terraplenagem e de supressão de vegetação nativa, dentre outras interferências.

\subsection{O estudo de alternativas no Processo $10214 / 2010$}

No Processo 10214/2010 foi realizado um EIA/Rima para a obtenção de Licença Prévia concomitantemente à Licença de Instalação para implantação da Estrada de Ligação entre as minas de Fábrica e do Pico, nos municípios de Ouro Preto e Itabirito-MG, respectivamente, tendo a empresa Vale como empreendedora. O objetivo principal deste projeto era reduzir o tráfego de caminhões devido à quantidade destes veículos observada nas rodovias BR-040 e BR-356, no trecho entre 
as cidades de Congonhas e Itabirito. Para definição das alternativas locacionais, consideraram-se as estradas existentes e os fatores ambientais e topográficos da região. Também se estudou a necessidade de movimentações de terra, como cortes e aterros e geração de material excedente, objetivando minimizar o volume de terraplanagem e das áreas impactadas, além de propor a alternativa mais viável economicamente. Assim, foram apresentadas quatro alternativas de traçado com diferentes rotas que serão descritas conforme as informações do EIA (Delphi Projetos e Gestão Ltda., 2010).

A primeira alternativa locacional possui uma extensão total de $36 \mathrm{~km}$ e contempla a utilização da estrada de terra existente, a qual interliga a mina do Pico à BR-040, passando pela mineração Herculano, com aproximadamente $13 \mathrm{~km}$ de extensão. Com esta proposta, haveria a necessidade de utilizar $23 \mathrm{~km}$ da BR-040, até a mina de Fábrica. Não haveria interferências consideráveis em novas áreas, mas, como a estrada existente possuía condições satisfatórias de tráfego, seriam realizadas melhorias gerais. Entretanto, o objetivo primordial do empreendimento, a diminuição do tráfego de caminhões na BR-040, não seria atendido.

A segunda alternativa possui uma extensão total de $28 \mathrm{~km}$ e se baseia em utilizar a estrada de terra que existe entre a mina do Pico e a BR-040, passando pela mineração Ponto Verde/Rede Gusa (Mineração Aredês), com aproximadamente $13 \mathrm{~km}$ de extensão. Segundo esta proposta, $15 \mathrm{~km}$ da BR040 até a mina de Fábrica seriam utilizados, o que faz com que a alternativa não cumpra com o objetivo central do projeto. Neste caso, seriam necessárias alterações em áreas de alta declividade da estrada de terra existente, sem citar a construção de uma nova ponte sobre um córrego para substituir a ponte de madeira deste local.
A alternativa III propõe a introdução de uma nova estrada realizada em área rural, totalizando 25 $\mathrm{km}$ de extensão. Seu traçado coincide em alguns pontos com a alternativa II e com a IV (descrita a seguir) e, do ponto de vista ambiental, interfere consideravelmente em áreas de mata nativa e, em menor proporção, na vegetação florestal ciliar do córrego do Silva, gerando, consequentemente, três transposições. A partir desta proposta, haveria a necessidade de obras de terraplanagem de grande influência, maior probabilidade de desestabilização de encostas e surgimento de processos erosivos.

A alternativa IV também possui traçado em área rural e aproximadamente $24 \mathrm{~km}$ de extensão. Em termos ambientais, esta proposta gera interferências, em sua maior parte, em áreas de campo nativo e, em menor intensidade, em vegetação florestal ciliar de afluentes e na transposição de um ribeirão. Grande extensão desta alternativa de traçado ocorre em Áreas de Preservação Permanente (APP) de topos de morro. Esta possibilidade é a que interfere em menor quantidade nos cursos d'água, necessitando apenas da transposição de dois ribeirões por meio de uma ponte e através de galeria e de diminutas drenagens pela construção de bueiros sob aterros. Além disso, parte do traçado está localizada em área já licenciada na mina do Pico.

Neste caso, para a escolha da melhor opção de traçado, as alternativas I e II foram descartadas por utilizarem trechos da BR-040, o que, consequentemente, não diminuiria o tráfego de caminhões e, assim, não atenderia o objetivo principal do projeto. Já a alternativa III foi considerada inviável pela necessidade de implantar interferências ao longo do traçado, como obras de terraplanagem com grande influência, as quais gerariam maior probabilidade de desestabilização de encostas e surgimento de processos erosivos. Desse modo, a alternativa IV foi escolhida, dentre outros pontos, por não utilizar a 
BR-040 e por sua menor extensão do traçado, equivalente a $24 \mathrm{~km}$. A proposta escolhida apresentava pouca interferência em cursos d'água e em vegetação florestal nativa, não gerava a necessidade de desapropriação de pessoas e, ainda, possuía parte do traçado em área já licenciada na mina do Pico. Após sua escolha, foram realizadas adequações simples em sua geometria, buscando reduzir o volume de corte e aterro, resultando assim no projeto final.

$\mathrm{Na}$ Tabela 3 é apresentado um resumo das alternativas propostas e a seleção daquelas que foram consideradas mais viáveis em cada um dos três estudos de caso, conforme informações dos respectivos EIA.

\subsection{Comparação dos estudos de caso com a literatura}

Baseado nos critérios escolhidos a partir da análise da literatura e conhecendo os casos com seus respectivos estudos de alternativas, a partir da análise de conteúdo, pôde-se realizar uma comparação entre ambos. A Tabela 4 apresenta a avaliação dos critérios gerais selecionados que devem ser utilizados na consideração de alternativas. Vale destacar que estes critérios são gerais, podendo ser devidamente adaptados para cada tipologia de empreendimento ou atividade.

Como apresentado anteriormente, todos os estudos de caso contavam com estudo de alternativas locacionais, sendo alguns mais bem detalhados e elaborados, enquanto outros eram mais simplificados, mas, de fato, os três casos analisados não apresentavam a deficiência apontada pelo MPF (2004), que é a ausência de proposição de alternativas. Cabe, então, avaliar como estas alternativas foram propostas e escolhidas.

É possível perceber pela Tabela 4 que sete dos 12 critérios foram cumpridos pelos três estudos de caso, sendo estes representados pelos números 1 , $2,3,6,7,11$ e 12. De acordo com as informações prestadas nos EIA/Rima, todos os estudos de alternativas tiveram foco em soluções e oportunidades

TABELA 3 - Estudo de alternativas nos estudos de caso apresentados

\begin{tabular}{|c|c|c|c|c|c|}
\hline \multirow{2}{*}{$\begin{array}{l}\text { Estudos de } \\
\text { Caso }\end{array}$} & \multicolumn{4}{|c|}{ Alternativas locacionais } & \multirow{2}{*}{$\begin{array}{c}\text { Alternativa } \\
\text { escolhida }\end{array}$} \\
\hline & $\mathbf{I}$ & II & III & IV & \\
\hline $\begin{array}{c}\text { Processo } \\
01323 / 2007\end{array}$ & $\begin{array}{c}\text { Compreende subtrechos } \\
01 \text { a } 08 \text { e incorpora parte } \\
\text { da rodovia MG-129; } \\
\text { influência negativa da } \\
\text { altimetria }\end{array}$ & $\begin{array}{c}\text { Compreende } \\
\text { subtrechos } 01 \text { a } 10 ; \\
\text { implantação de obras } \\
\text { em nível elevado } \\
\text { devido a áreas } \\
\text { sensíveis ao ambiente }\end{array}$ & $\begin{array}{c}\text { Alargamento da } \\
\text { rodovia atual entre } \\
\text { Belo Horizonte } \\
\text { e Itabira, mais } \\
\text { modificações } \\
\text { localizadas }\end{array}$ & - & $\begin{array}{l}\text { II, com alguns } \\
\text { aspectos da } \\
\text { alternativa III }\end{array}$ \\
\hline $\begin{array}{c}\text { Processo } \\
19754 / 2009\end{array}$ & $\begin{array}{c}\text { Traçado variável entre } \\
\text { os dois lados da pista, } \\
\text { gerando menores } \\
\text { impactos e interferências }\end{array}$ & Não implantação & ( & - & I \\
\hline $\begin{array}{c}\text { Processo } \\
10214 / 2010\end{array}$ & $\begin{array}{l}\text { Utilizar estrada de terra } \\
\text { existente acrescida de } \\
13 \mathrm{~km} \text { de determinado } \\
\text { trecho da BR-040 }\end{array}$ & $\begin{array}{c}\text { Utilizar estrada } \\
\text { de terra existente } \\
\text { acrescida de } 15 \mathrm{~km} \text { de } \\
\text { determinado trecho da } \\
\text { BR-040 }\end{array}$ & $\begin{array}{l}\text { Nova estrada de } \\
\text { terra com } 25 \mathrm{~km}, \\
\text { influenciando em } \\
\text { mata nativa }\end{array}$ & $\begin{array}{l}\text { Nova estrada de } \\
\text { terra com } 24 \mathrm{~km} \text {, } \\
\text { gerando menores } \\
\text { impactos; parte } \\
\text { do traçado já } \\
\text { licenciado }\end{array}$ & IV \\
\hline
\end{tabular}


TABELA 4 - Avaliação dos estudos de alternativas dos estudos de caso selecionados

\section{Critérios Gerais}

Processo
$01323 / 2007$

Processo
$19754 / 2009$

Processo

1 - Foco em soluções e oportunidades

2 - Introdução de conceitos de sustentabilidade e capacidade suporte do ambiente

3 - Incentivo à inovação e prevenção

4 - Ser rigoroso, objetivo e não enviesado

5 - Ponderar sobre a alternativa de não ação

6 - Embasar em dados confiáveis e atualizados

7 - Ocorrer antes da tomada de decisões importantes

8 - Possibilitar que todos os interessados possam participar de forma eficaz

$\begin{array}{lll}\checkmark & \checkmark & \checkmark \\ \checkmark & \checkmark & \checkmark \\ \checkmark & \checkmark & x \\ x & x & x \\ x & \checkmark & \checkmark \\ \checkmark & \checkmark & x \\ \checkmark & \checkmark & x \\ x & x & \checkmark \\ \checkmark & \checkmark & \checkmark \\ \checkmark & x & \checkmark \\ \checkmark & \checkmark & \end{array}$

9 - Propor escolhas realistas evitando a elaboração arbitrária de alternativas, incluindo propostas inviáveis apenas para engrandecer o estudo

10 - Justificar e analisar de forma simples os motivos que eliminaram alguma alternativa e o processo de escolha

11 - Evitar a construção de "problemas" para restringir possíveis

"soluções" (alternativas)

12 - Evitar favorecer alternativas utilizadas anteriormente, desconsiderando opções modernas

$\checkmark$ : Critérios cumpridos; $X$ : Critérios não cumpridos.

(critério 1) por otimizarem seus custos operacionais, seja adotando menores trajetos ou por utilizar faixas existentes, terrenos das próprias empresas ou licenciamentos já realizados. Quanto ao critério 2, as propostas pareceram buscar introduzir conceitos de sustentabilidade e capacidade suporte do ambiente, optando por evitar a degradação ou escolher áreas já degradadas por meio de desvios de ecossistemas sensíveis, proximidade com nascentes, margens de córregos e vegetação nativa e pela seleção de técnicas que impactem menos. $\mathrm{O}$ critério 3 diz respeito ao incentivo à inovação e à prevenção, surgindo nos estudos ao requisitar a segurança na operação das rodovias e por evitar custos com terraplanagem e suas consequências (bota-fora e aproveitamento).

Dois critérios importantes para almejar o objetivo da AIA, representados pelos números 6 e 7, foram também abordados por todos os estudos, representando trabalhos realizados com dados confiáveis e atualizados e a tomada de decisões importantes baseada nas alternativas propostas. Por fim, dos critérios bem avaliados, os critérios 11 e 12 foram considerados nas propostas, respectivamente, por não haver nenhum estudo de caso em que haja a construção de "problemas" para restringir possíveis "soluções" e por não desconsiderarem 
opções modernas para favorecer possibilidades já utilizadas. Esta última pode ser confirmada pela escolha realizada no Processo 01323/2007, onde ocorreu a junção de duas alternativas para criar uma opção mais atual e capaz de solucionar o problema exposto.

Mesmo com os estudos tendo sido bem avaliados em sete critérios, é pertinente destacar que critérios fundamentais de boas práticas para $o$ desenvolvimento do estudo de alternativas foram deixados de lado. Estes pontos e sua importância para o contexto da AIA serão discutidos a seguir.

Quanto à avaliação de não cumprimento, não foram abordados em nenhum dos processos os critérios 4 e 8 . O primeiro (critério 4), representado pela necessidade do estudo de alternativas ser rigoroso, objetivo e não enviesado, não foi encontrado nos estudos de caso devido à falta de detalhamento, apresentando alternativas superficiais e sem a real demonstração de seus impactos. O segundo (critério 8) é referente à participação dos interessados, fato que aparentemente não foi realizado nas três propostas, uma vez que não existiu no EIA nenhum apontamento de que o processo de seleção de alternativas envolveu a participação dos interessados, principalmente dos afetados pelo projeto. É pertinente que a participação ocorra o mais cedo possível na AIA (IAIA, 1999), começando na pré-avaliação e continuando por todo o processo (Vasconcelos et al., 2000), inclusive durante o estudo de alternativas. Isso devido ao fato de que em seus vários estágios a participação fornece oportunidades para o envolvimento e a negociação entre os diferentes atores (Devlin \& Yap, 2008).

Quanto aos pontos restantes em que existiu diferença de avaliação entre os casos, o critério 5 (ponderar sobre a alternativa de não ação) foi abordado apenas pelo Processo 19754/2009, sendo não cumprido nos demais. Esta opção de não ação é extremamente importante por mostrar que realizar o projeto é realmente necessário. Além disso, vem de encontro ao estabelecido na Resolução Conama $n^{\circ}$ 01 de 1986, que estabelece entre as diretrizes gerais para a elaboração do EIA que este deve conter o estudo de alternativas tecnológicas e locacionais, confrontando as alternativas de execução com a hipótese de não execução do projeto (CONAMA, 1986). Em outras palavras, dois dos processos analisados não cumpriram com as diretrizes estabelecidas pela legislação ambiental.

O critério 9, que trata da elaboração arbitrária de alternativas que geram a inclusão de propostas inviáveis apenas para engrandecer o estudo, não foi cumprido no Processo 10214/2010. Neste caso, duas alternativas foram criadas de forma a serem automaticamente descartadas, pois não cumpriam o objetivo central do projeto. Isso retrata a deficiência de apresentação de alternativas reconhecidamente inferiores à selecionada, já destacada pelo MPF (2004).

O último aspecto a ser discutido em relação à avaliação trata-se do critério 10 , que relata sobre a exclusão sem justificativa de alternativas consideráveis, antes de avaliá-las detalhadamente. Este ponto ocorreu no Processo 19754/2009, onde foi citada a possibilidade de se implantar a obra no sentido direito ou esquerdo da pista, mas estas opções não são apresentadas, surgindo em seu lugar uma alternativa que contemplou uma proposta variável entre os dois casos. Vale ressaltar que, embora a alternativa que une as duas possibilidades pareça ser realmente a mais viável e, por consequência, tenha sido a escolhida, deveria ser explicitado no estudo o detalhamento dos outros dois caminhos e o porquê de sua exclusão, para que não ocorra a falha apontada por Zubair (2001), que é a escolha de alternativas ambientalmente inviáveis. 
Apesar de os três casos terem apresentado estudo de alternativas e terem cumprido vários critérios analisados, exibiram falhas graves que comprometeram a qualidade da avaliação realizada. De modo geral, não foi deixado claro que a escolha realizada realmente era a ambientalmente mais adequada e não teve nenhuma forma de participação dos afetados e interessados, sendo feita pelo próprio empreendedor. Este fato pode acarretar decisões com pouca ou nenhuma análise ambiental (Lisboa, 2002).

Outro fator a ser considerado e que foi notado em todos os casos analisados - apesar de não ter constituído um critério de análise, mas ter relação com o critério 4 (ser rigoroso, objetivo e não enviesado) e já ter sido apontado como falha pelo levantamento do MPF (2004) - é o domínio dos fatores econômicos sobre os ambientais na adoção e na escolha de alternativas. No Processo 01323/2007, o EIA aponta que para a escolha da alternativa considerou-se o fato de que custos ambientais consequentes da instalação da proposta seriam assimilados pelos benefícios sociais e econômicos intrínsecos ao projeto (CEC, 2007). No Processo 19754/2009, é relatado que a rodovia, por receber incentivos do PAC, já possui viabilidade em termos econômicos (Escritório Técnico H. Lisboa da Cunha, 2009). E no Processo 10214/2010, a alternativa IV foi escolhida, dentre outros pontos, por não utilizar a BR-040 e por sua menor extensão do traçado (Delphi Projetos e Gestão Ltda., 2010). Assim, não possui efetividade um estudo de alternativas que, embora realizado e que atenda alguns critérios de boas práticas, não cumpre seu papel de procurar uma alternativa mais sustentável e com menor potencial de causar impactos negativos (Oliveira, 2004; Paliwal, 2006) e propiciar o uso mais racional dos recursos ambientais.

Para tentar entender uma possível origem para a falha no estudo de alternativas, foi anali- sado o Termo de Referência (TR) disponibilizado pelo órgão ambiental mineiro para a elaboração dos EIA/Rima. Ao consultar a página do órgão ambiental (http://www.meioambiente.mg.gov.br/ noticias/1/1167-termos-de-referencia-para-elaboracao-de-estudo-de-impactorelatorio-de-impacto-ambiental-eiarima), foi verificado que não existe um TR específico para rodovias, devendo ser usado o TR geral. Este, no que diz respeito a alternativas, solicita apenas "Apresentar também esclarecimentos sobre as possíveis alternativas tecnológicas e/ou locacionais, inclusive aquelas de não se proceder à sua implantação" (FEAM, 2017). Assim, as falhas nos estudos ambientais podem estar associadas às falhas em etapas anteriores do processo de AIA, como a de escopo, conforme já foi apontado por Almeida et al. (2014) ao verificar a baixa qualidade de Relatórios de Controle Ambiental apresentados ao órgão ambiental mineiro.

Finalmente, vale destacar que, conforme apontado por Pope et al. (2013), a consideração de alternativas para o projeto está entre as áreas da AIA que universalmente persistem em ter uma prática deficiente. Neste contexto, para amenizar esta deficiência, torna-se fundamental a integração da AIA com instrumentos de planejamento, como o zoneamento ambiental (Oliveira, 2004), cabendo estudos futuros para que instrumentos de planejamento, como o zoneamento, sejam incluídos e auxiliem no estudo e na escolha de alternativas não só de rodovias, mas de todas as tipologias de atividades.

\section{Considerações finais}

A análise de alternativas locacionais, mesmo sendo de extrema importância e relevância dentro de um Estudo de Impacto Ambiental, foi preca- 
riamente observada nos três casos analisados. De forma geral, os estudos apresentaram-se falhos por não abordarem satisfatoriamente critérios básicos apresentados pela literatura, além de exibirem falhas primárias, como a proposição de alternativas já de início descartáveis, e possuírem uma explicação muito simplificada da escolha realizada. Além disso, foi dada mais importância a fatores de cunho econômico para a escolha da alternativa, mesmo tendo o Estudo de Impacto Ambiental a função de discussão da viabilidade ambiental.

Sendo assim, baseando-se nas análises aqui realizadas, pode-se dizer que no território mineiro o estudo de alternativas locacionais de rodovias é frágil, não havendo a consideração necessária e devida deste fator imprescindível na tomada de decisões. Portanto, há a necessidade de aperfeiçoamentos que podem começar pela elaboração de TR com exigências mais criteriosas sobre o estudo de alternativas, inclusive para cumprir o exigido na Resolução Conama no 001/1986. Além disso, deve haver uma maior exigência do órgão ambiental quanto ao estudo de alternativas, pois se ele aceita e aprova estudos ruins, não exigindo nada melhor, os EIA vão continuar sendo problemáticos para a discussão da viabilidade ambiental dos empreendimentos.

Por fim, a presente pesquisa possui limitações por ter analisado somente as informações contidas no EIA e não o processo no qual se deu o desenvolvimento do estudo de alternativas, além de não ter conseguido levantar indicadores específicos para a análise do traçado de rodovias. Em termos numéricos, a limitação ocorre em relação ao trabalho ter analisado somente três casos, mesmo estes sendo a totalidade de processos de licenciamento de rodovias apoiados na elaboração de EIA disponíveis no SIAM. No entanto, estas limitações não alteram a conclusão da pesquisa de que os estudos de alternativas para traçado de rodovias realizados no âmbito do licenciamento estadual de Minas Gerais são falhos. Estudos futuros, englobando mais processos e mais tipologias de empreendimentos, podem complementar os resultados aqui encontrados, de forma a caracterizar de modo mais abrangente o estudo de alternativas nos processos de licenciamento tramitados no órgão ambiental estadual de Minas Gerais.

\section{Referências}

ABEMA - Associação Brasileira de Entidades Estaduais de Meio Ambiente. Novas propostas para o licenciamento ambiental no Brasil. Brasília: Associação Brasileira de Entidades Estaduais de Meio Ambiente - ABEMA, 2013. 92p.

Agra Filho, S. S.; Marinho, M. M. O.; Santos, J. O. Avaliação de Impacto Ambiental (AIA): uma proposta metodológica para análise de efetividade de aplicação através da avaliação Ex-Post. In: Anais do $24^{\circ}$ Congresso Brasileiro de Engenharia Sanitária e Ambiental. Belo Horizonte, 2007.

Agra Filho, S. S.; Marinho, M. M. O.; Orrico, S. R. M.; Santos, F. C. Avaliação de Impactos Ambientais: uma dis- cussão dos procedimentos metodológicos da aplicação no estado da Bahia. In: Anais do $1^{\circ}$ Congresso Brasileiro de Avaliação de Impacto. São Paulo, 2012.

Almeida, M. R. R.; Alvarenga, M. I. N.; Cespedes, J. G. Geociencias, 33(1), 106-118, 2014.

Almeida, M. R. R.; Montaño, M. Benchmarking na avaliação de impacto ambiental: O sistema mineiro frente às melhores práticas internacionais. Sociedade \& Natureza, 27(1), 81-96, 2015.

Almeida, M. R. R.; Montaño, M. A efetividade dos sistemas de Avaliação de Impacto Ambiental nos estados de 
São Paulo e Minas Gerais. Ambiente \& Sociedade, 20(2), 79-106, 2017.

Bandeira, C.; Floriano, E. P. Avaliação de impacto ambiental de rodovias. 1. ed. Santa Rosa, 2004. (Caderno Didático n. 8)

Bardin, L. Análise de conteúdo. São Paulo: Edições 70, 2011.

Canter, L. W. Environmental Impact Assessment. 2. ed. New York: McGraw-Hill, 1996.

CEC - Consórcio Consol/Enecon/Contécnica. Estudo de Impacto Ambiental do Projeto de Ampliação da Capacidade e Modernização da Ligação Rodoviária entre Belo Horizonte e Governador Valadares - Plano Funcional. Belo Horizonte, 2007.

CONAMA - Conselho Nacional do Meio Ambiente (Brasil). Resolução $n^{\circ}$ 001, de 23 de janeiro de 1986. Brasília: DOU de 17/2/1986.

Delphi Projetos e Gestão Ltda. Estudo de Impacto Ambiental da implantação da Estrada de Ligação das Minas do Pico a Fábrica. Belo Horizonte, 2010.

Desmond, M. Identification and development of waste management alternatives for Strategic Environmental Assessment (SEA). Environmental Impact Assessment Review, 29, 51-59, 2009.

Devlin, J. F.; Yap, N. T. Contentious politics in environmental assessment: blocked projects and winning coalitions. Impact Assessment and Project Appraisal, 26(1), 17-27, 2008. doi: 10.3152/146155108X279939

Duarte, C. G.; Dibo, A. P. A.; Sánchez, L. E. O que diz a pesquisa acadêmica sobre avaliação de impacto e licenciamento ambiental no Brasil? Ambiente \& Sociedade, 20(1), 245-278, 2017.

Duarte, C. G.; Dibo, A. P. A.; Siqueira-Gay, J.; Sánchez, L. E. Practitioners' perceptions of the Brazilian environmental impact assessment system: results from a survey. Impact Assessment and Project Appraisal, 35(4), 1-17, 2017.

Escritório Técnico H. Lisboa da Cunha. EIA/RIMA Projeto de Restauração, Adequação e Duplicação da Rodovia BR050, no subtrecho entre a Divisa GO/MG e o Entroncamento com as Rodovias BR 65/452/455/497, no Estado de Minas Gerais. Rio de Janeiro, 2009.

FEAM - Fundação Estadual de Meio Ambiente. Termos de Referências. Disponível em: <http://www.feam.br/images/ stories/arquivos/tr/eia_rima_geral001.pdf $>$. Acesso em: out. 2017.

Fonseca, A.; Sánchez, L. E.; Ribeiro, J. C. J. Reforming EIA systems: A critical review of proposals in Brazil. Environmental Impact Assessment Review, 62, 90-97, 2017.

Furlanetto, T. Estudo de alternativas locacionais para a viabilidade ambiental de empreendimentos: o caso do aeroporto de Ribeirão Preto - SP. São Carlos, Dissertação (Mestrado em Ciências da Engenharia Ambiental) - USP, 2012.

Governo de Minas. Rodovias. Disponível em: <http:// mg.gov.br/conheca-minas/rodovias $>$. Acesso em: out. 2017.

Hickie, D.; Wade, M. Development of guidelines for improving the effectiveness of environmental assessment. Environmental Impact Assessment Review, 18, 267-287, 1998.

IAIA - International Association for Impact Assessment. Principles of Environmental Impact Assessment Best Practice.Fargo: IAIA, Special Publication v.1, 1999. Disponível em: <http://www.iaia.org/publicdocuments/ special-publications/Principles\%20of\%20IA_web.pdf>. Acesso em: jan. 2016.

IAIA - International Association for Impact Assessment. FASTIP:Alternatives inProject EIA. 2015. Disponível em: $<$ https://www.iaia.org/uploads/pdf/FasTips_11_AlternativesinProjectEIA.pdf > . Acesso em: out. 2017.

Jay, S.; Jones, C.; Slinn, P.; Wood, C. Environmental impact assessment: Retrospect and prospect. Environmental Impact Assessment Review, 27(4), 287-300, 2007. doi: 10.1016/j. eiar.2006.12.001

Kruopienè, J.; Židonienè, S.; Dvarionienè, J. Current practice and shortcomings of EIA in Lithuania. Impact Assessment and Project Appraisal, 29, 305-309, 2009. doi: 10.1016/j.eiar.2009.02.003

Kvaerner, J.; Swensen, G.; Erikstad, L. Assessing environmental vulnerability in EIA - the content and context of the vulnerability concept in an alternative approach to standard EIA procedure. Environmental Impact Assessment Review, 26, 511-527, 2006.

Lisboa, M. V. Contribuição para tomada de decisão na classificação e seleção de alternativas de traçado para rodovias em trechos urbanizados. São Carlos, Dissertação (Mestrado em Engenharia de Transportes) - USP, 2002. 
Lobos, V.; Partidário, M. Theory versus practice in Strategic EnvironmentalAssessment (SEA). Environmental Impact Assessment Review, 48, 34-46, 2014.

Morgan, R. K. Environmental impact assessment: the state of the art. Impact Assessment and Project Appraisal, 30(1), 5-14, 2012. doi: 10.1080/14615517.2012.661557

MPF - Ministério Público Federal. Deficiências em Estudos de Impacto Ambiental: síntese de uma experiência. Brasília: $4^{\text {a }}$ Câmara de Coordenação e Revisão, Escola Superior do Ministério Público da União, 2004.

Oliveira, I. S. D. A contribuição do zoneamento ecológico econômico na avaliação de impacto ambiental: bases e propostas conceituais. São Carlos, Dissertação (Mestrado em Ciências da Engenharia Ambiental) - USP, 2004.

Omena, M. L. R. A.; Santos, E. B. Análise da efetividade da Avaliação de Impactos Ambientais - AIA - da Rodovia SE 100/Sul-Sergipe. Revista Brasileira de Gestão e Desenvolvimento Regional, 4(1), 221-237, 2008.

Paliwal, R. EIA practice in India and its evaluation using SWOT analysis. Environmental Impact Assessment Review, 26, 492-510, 2006.doi:10.1016/j.eiar.2006.01.004

Pinho, P.; Maia, R.; Monterroso, A. The quality of Portuguese Environmental Impact Studies: the case of small hydropower projects. Environmental Impact Assessment Review, 27, 189-205, 2007. doi: 10.1016/j.eiar.2006.10.005

Pope, J.; Bond, A.; Morrison-Saunders, A.; Retief, F. Advancing the theory and practice of impact assessment: Setting the research agenda. Environmental Impact Assessment Review, 41, 1-9, 2013. Disponível em: <http://dx.doi. org/10.1016/j.eiar.2013.01.008>.

Prado Filho, J. F.; Souza, M. P. O Licenciamento Ambiental da mineração no Quadrilátero Ferrífero de Minas Gerais: uma análise da implementação de medidas de controle am- biental formuladas em EIAS/RIMAS. Engenharia Sanitária e Ambiental, 9(4), 343-349, 2004.

Rangel, T. A. C.; Bueno, C. Impacto dos Empreendimentos Linearesna Paisagem. Revista Interdisciplinar UVA, 15, 80-98, 2016.

Sá-Silva, J. R.; Almeida, C. D.; Guindani, J. F. Pesquisa documental: pistas teóricas e metodológicas. Revista Brasileira de História \& Ciências Sociais, 1(1), 1-15, 2009.

Sánchez, L. E. Avaliação de impacto ambiental: conceitos e métodos. São Paulo: Oficina de Textos, 2008. 495p.

Smith, M. D. A review of recent NEPA alternatives analysis case law. Environmental Impact Assessment Review, 27, 126-144, 2007.

Steinemann, A. Improving alternatives for environmental impact assessment. Environmental Impact Assessment Review, 21, 3-21, 2001.

Tickener, J. A.; Geiser, K. The precautionary principle stimulus for solutions - and alternatives - based environmental policy. Environmental Impact Assessment Review, 24, 801-824, 2004.

Vasconcelos, C.; Hamilton, A.; Barrett, P. Public participation in EIA: A study from a Portuguese perspective. Journal of Environmental Assessment Policy and Management, 2(4), 561-582, 2000.

Wood, C. Environmental impact assessment: a comparative review. Harlow: Prentice Hall, 2002.

Yentzer, D. A.; Clarck, R. The "Hierarchy of needs" Screening Process: A method for rapid development and rationalization of reasonable alternatives. Environmental Practice, 8, 215-217, 2006.

Zubair, L. Challenges for environmental impact assessment in Sri Lanka. Environmental Impact Assessment Review, 21(5), 469-478, 2001. 